\title{
Modulation of calcification of vascular smooth muscle cells in culture by calcium antagonists, statins, and their combination
}

\author{
Astrid Trion • Cindy Schutte-Bart • \\ Wilhelmina H. Bax · J. Wouter Jukema • \\ Arnoud van der Laarse
}

Received: 9 June 2007 / Accepted: 13 September 2007/Published online: 2 October 2007

(C) Springer Science+Business Media, LLC. 2007

\begin{abstract}
Background Vascular calcification is an organized process in which vascular smooth muscle cells (VSMCs) are implicated primarily. The purpose of the present study was to assess the effects of calcium antagonists and statins on VSMC calcification in vitro. Methods VSMC calcification was stimulated by incubation in growth medium supplemented with $10 \mathrm{mmol} / \mathrm{l} \beta$-glycerophosphate, $8 \mathrm{mmol} / 1 \mathrm{CaCl}_{2}, 10 \mathrm{mmol} / \mathrm{l}$ sodium pyruvate, $1 \mu \mathrm{mol} / \mathrm{l}$ insulin, $50 \mu \mathrm{g} / \mathrm{ml}$ ascorbic acid, and $100 \mathrm{nmol} / \mathrm{l}$ dexamethasone (calcification medium). Calcification, proliferation, and apoptosis of VSMCs were quantified. Results Calcium deposition was stimulated dose-dependently by $\beta$-glycerophosphate, $\mathrm{CaCl}_{2}$, and ascorbic acid (all $P<0.01)$. Addition of amlodipine $(0.01-1 \mu \mathrm{mol} / \mathrm{l})$ to the calcification medium did not affect VSMC calcification. However, atorvastatin $(2-50 \mu \mathrm{mol} / \mathrm{l})$ stimulated calcium deposition dose-dependently. Combining treatments stimulated calcification to a degree similar to that observed with atorvastatin alone. Both atorvastatin and amlodipine inhibited VSMC proliferation at the highest concentration used. Only atorvastatin $(50 \mu \mathrm{mol} / \mathrm{l})$ induced considerable apoptosis of VSMCs. Conclusion In vitro calcification of VSMCs is not affected by amlodipine, but is stimulated by atorvastatin at concentrations $\geq 10 \mu \mathrm{mol} / 1$, which could contribute to the plaque-stabilizing effect reported for statins.
\end{abstract}

J. W. Jukema is an Established Clinical Investigator of The Netherlands Heart Foundation 2001D032.

A. Trion · C. Schutte-Bart - W. H. Bax .

J. W. Jukema - A. van der Laarse $(\square)$

Department of Cardiology, Leiden University Medical Center,

Albinusdreef 2, Leiden 2333 ZA, The Netherlands

e-mail: a.van_der_laarse@lumc.nl
Keywords Calcification - Atherosclerosis · Vascular smooth muscle cells · In vitro model . Calcium antagonist $\cdot$ Statin

\section{Introduction}

Atherosclerosis is the principal cause of coronary artery disease, stroke, and peripheral artery disease, and is the major cause of mortality in the Western hemisphere. Vascular calcification is a prominent feature of atherosclerosis, and it is associated with an increased risk of myocardial infarction [1]. Vascular calcification refers to the deposition of calcium phosphate mineral, most often in the form of hydroxyapatite, in the vessel wall. Calcification of the vessel wall and heart valves is associated with ageing, diabetes, and uremia [2-4].

Vascular calcification is now considered to be an organized, regulated process comparable to bone mineralization. The presence of various components associated with bone mineralization, such as bone morphogenetic protein, osteocalcin, osteopontin, osteoblast-like cells, and matrix vesicles in atherosclerotic lesions supports this concept [5-7]. Vascular cells such as vascular smooth muscle cells (VSMCs) and pericyte-like cells play an important role in vascular calcification [8].

The lipophilic calcium antagonist (CA) amlodipine has been shown to restore cholesterol-induced membrane bilayer abnormalities in VSMCs derived from the atherosclerotic rabbit aorta [9, 10], thereby restoring normal calcium homeostasis. Other mechanisms, through which CAs may affect atherosclerosis development include inhibition of proliferation and migration of VSMCs [11, 12], and inhibition of lipoprotein oxidation [13]. In addition, CAs modify binding of monocytes to the 
endothelium, and activate synthesis of matrix components [14]. The effects of CAs on atherosclerotic calcification have not been widely studied, however.

Lipids may contribute to atherosclerotic calcification $[15,16]$. Recently statins have been shown to decrease the progression of coronary artery calcification and aortic valve calcification $[17,18]$. Therapy with a combination of a CA and a statin might be more atheroprotective than either treatment alone [19, 20]. Combination therapy with CA and statin was shown to improve endothelial function and arterial compliance, to diminish LDL atherogenicity, and to slow the progression of atherosclerosis. So far, the effects of the combination therapy with CA and statin on vascular calcification have not been studied extensively.

We used an in vitro model of vascular calcification by neonatal rat VSMCs, isolated by outgrowth from aortic explants. The purpose of this investigation was to identify the factors, and their concentrations, that are mandatory to induce calcification of aortic VSMCs in vitro. Since calcium is such a major component of advanced atherosclerotic lesions, we furthermore studied whether the CA amlodipine and the statin atorvastatin, alone and in combination, affected calcification of aortic VSMCs in vitro.

\section{Materials and methods}

\section{Cell culture}

Vascular smooth muscle cells (VSMCs) were obtained from segments of aortas explanted from 2-day old Wistar rats. Aortic segments were obtained aseptically and cut open longitudinally. The endothelium was removed by gently rubbing the luminal side of the aortas over the surface of a tissue culture dish (Falcon). Subsequently, the aortas were placed, lumen side down, on the bottom of a tissue culture flask (Greiner), and allowed to adhere for approximately $3 \mathrm{~h}$. Then tissues were immersed in growth medium consisting of Dulbecco's modified Eagle's medium (DMEM) (Life Technologies) supplemented with $10 \%$ heat-inactivated fetal bovine serum (FBS) (Life Technologies), penicillin (100 U/ml), and streptomycin $(100 \mu \mathrm{g} / \mathrm{ml})$ (both supplied by BioWhittaker Europe). Seven days later the aortic tissues were removed, and the VSMCs that had grown out were detached by trypsinization. The detached cells were resuspended in growth medium and seeded in tissue culture flasks (Greiner), 6 or 12-well plates or on glass cover slips.

\section{Immunocytochemistry}

Subconfluent cultures at early passage were examined for the presence of $\alpha$-smooth muscle actin, SM myosin heavy chain and calponin using specific antibodies (anti-SM actin clone 1A4 (1:400), Sigma; anti-SM myosin heavy chain (1:100), SanverTech; anti-calponin (1:10,000), Sigma). After fixation in $1 \%$ formalin in PBS on ice for $30 \mathrm{~min}$, cells were permeabilized with $0.1 \%$ Triton $\mathrm{X}-100$ for $30 \mathrm{~min}$, and subsequently incubated with the primary antibody for several hours at $4^{\circ} \mathrm{C}$. Secondary antibodies were conjugated with fluorescein isothiocyanate (FITC) or Cy3 (Sigma). To identify cell nuclei, the cells were also stained with Hoechst 33342 (10 $\mu \mathrm{g} / \mathrm{ml}$; Molecular Probes). Immunofluorescent images were obtained using a fluorescence microscope (Nikon Eclipse) equipped with $20 \times$, 40×, and $100 \times$ objectives and a digital camera (Nikon DXM1200).

In vitro calcification of VSMCs

Calcification of VSMC cultures was induced by the method of Shioi et al. [21] with minor modifications. When confluent, the incubation medium of VSMCs was switched from growth medium to calcification medium. Calcification medium consisted of DMEM (high glucose, $4.5 \mathrm{~g} / \mathrm{l}$ ) supplemented with $15 \% \mathrm{FBS}$, penicillin $(100 \mathrm{U} / \mathrm{ml})$, streptomycin $(100 \mu \mathrm{g} / \mathrm{ml}), 8 \mathrm{mmol} / \mathrm{l} \mathrm{CaCl}_{2}, 10 \mathrm{mmol} / \mathrm{l}$ sodium pyruvate, $1 \mu \mathrm{mol} / \mathrm{l}$ insulin, $50 \mu \mathrm{g} / \mathrm{ml}$ ascorbic acid, $10 \mathrm{mmol} / \mathrm{l} \beta$-glycerophosphate, and $100 \mathrm{nmol} / \mathrm{l}$ dexamethasone. The medium was replaced with fresh medium every 2-3 days. In several experiments, the concentrations of $\mathrm{CaCl}_{2}$, ascorbic acid, $\beta$-glycerophosphate, and dexamethasone were varied.

In another series of experiments, calcification medium was supplemented with amlodipine $(0.01-1 \mu \mathrm{mol} / \mathrm{l})$, atorvastatin $(2-50 \mu \mathrm{mol} / \mathrm{l})$, or a combination of these drugs. The CA amlodipine was dissolved in absolute ethanol at a concentration of $1 \mathrm{mmol} / \mathrm{l}$. This stock solution was to be diluted at least 1:1,000 in culture medium. Atorvastatin, an inhibitor of HMG-CoA reductase, was dissolved in absolute ethanol at a concentration of $10 \mathrm{mmol} / \mathrm{l}$. This stock solution was to be diluted at least 1:200 in culture medium.

Cells treated with calcification medium without added drugs were used as controls. Appropriate amounts of solvent (ethanol) were added to these controls. Addition of ethanol at these levels did not affect cell growth. After 3 weeks of incubation, calcification was quantified.

Assessment of calcium deposition

VSMCs were decalcified with $0.6 \mathrm{~N} \mathrm{HCl}$ for $24 \mathrm{~h}$. The calcium contents of the supernatants were determined by spectrophotometer using the $o$-cresolphthalein method (Roche Diagnostics). After decalcification, the cells were washed with PBS and scraped from the culture plate. The 
protein content was measured using the BCA protein assay (Pierce). The calcium content of the cell layer was normalized to protein content.

\section{Proliferation assay}

VSMCs were seeded at a density of $5 \times 10^{3}-2 \times 10^{4}$ cells/ well in a 96-well plate and allowed to attach overnight. Subsequently, the standard culture medium (DMEM, 10\% FBS, antibiotics) was replaced by calcification medium supplemented with amlodipine, atorvastatin, or a combination of both. Cell proliferation was assessed at day 4 and day 9 using the Cell Proliferation Kit II (XTT, Roche), which is a colorimetric assay for the non-radioactive quantification of cell proliferation and viability.

\section{Detection of apoptosis}

To identify apoptotic cells by assessment of condensation of nuclear chromatin, VSMC cultures were stained with Hoechst $33342(10 \mu \mathrm{g} / \mathrm{ml}$; Molecular Probes) for $10 \mathrm{~min}$ in the dark. Immunofluorescent images were obtained using a fluorescence microscope (Nikon Eclipse) equipped with 20x, 40x, and 100x objectives and a digital camera (Nikon DXM1200). In 6 frames per treatment, an average of 250 nuclei per frame were assessed for the presence of apoptosis.

\section{Statistical analysis}

Results are expressed as mean \pm SEM. For statistical analysis, SPSS 10.0 for Windows was used. Since the data were not normally distributed, non-parametric tests were used for comparisons between groups. Overall comparisons between groups were performed with the Kruskall-Wallis test. If only two groups were compared, Mann-Whitney rank sum tests were used. $P$ values less than 0.05 were regarded as significant.

\section{Results}

VSMCs that have been cultured from rat aortic explants for approximately 7 days stained positive for $\alpha$-smooth muscle-actin, smooth muscle myosin heavy chain, and calponin, confirming the smooth muscle cell nature of these cells (Fig. 1, online supplement). VSMCs of passage 3-6 were subsequently used in calcification experiments.

Calcium deposition and dependence of extracellular $\mathrm{Ca}^{2+}$ concentration

Cells were incubated with calcification medium supplemented with various $\mathrm{Ca}^{2+}$ concentrations for 21 days. At $\mathrm{Ca}^{2+}$ concentrations $\leq 3 \mathrm{mmol} / 1$, hardly any calcium deposition was observed, but at $\mathrm{Ca}^{2+}$ concentrations $>3 \mathrm{mmol} / \mathrm{l}$, a dose-dependent increase in calcium deposition was observed (Fig. 2A). The amount of calcium deposition was positively correlated to calcium concentration in the culture medium $(P<0.01)$. We chose to continue with $8 \mathrm{mmol} / \mathrm{l}$ of $\mathrm{Ca}^{2+}$-ions added to the calcification medium.

Calcium deposition and dependence of extracellular phosphate concentration

Shioi et al. have demonstrated that $\beta$-glycerophosphate accelerates in vitro calcification of VSMCs and induces extensive calcium deposition in a manner analogous to in vitro mineralization by osteoblasts [21]. In our model of
Fig. 1 (A) Rat aortic VSMC isolated by outgrowth, phase contrast. Immunofluorescent staining of neonatal rat VSMC incubated with (B) (and insert) anti-smooth muscle actin $\mathrm{Ab}$, (C) anti-smooth muscle myosin $\mathrm{Ab}$, and (D) anti-calponin $\mathrm{Ab}$. Original magnification $\mathrm{B}$ and $\mathrm{C}$ $\times 100, D$ and insert $\times 200$. VSMC = vascular smooth muscle cell
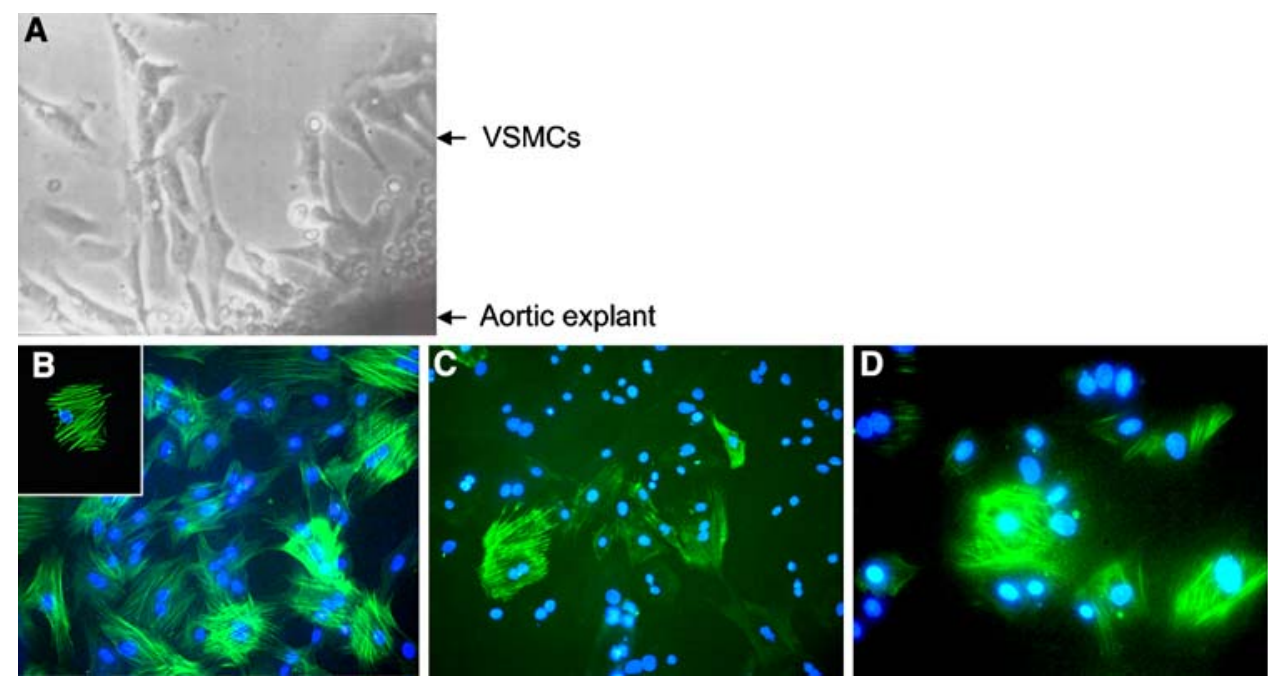

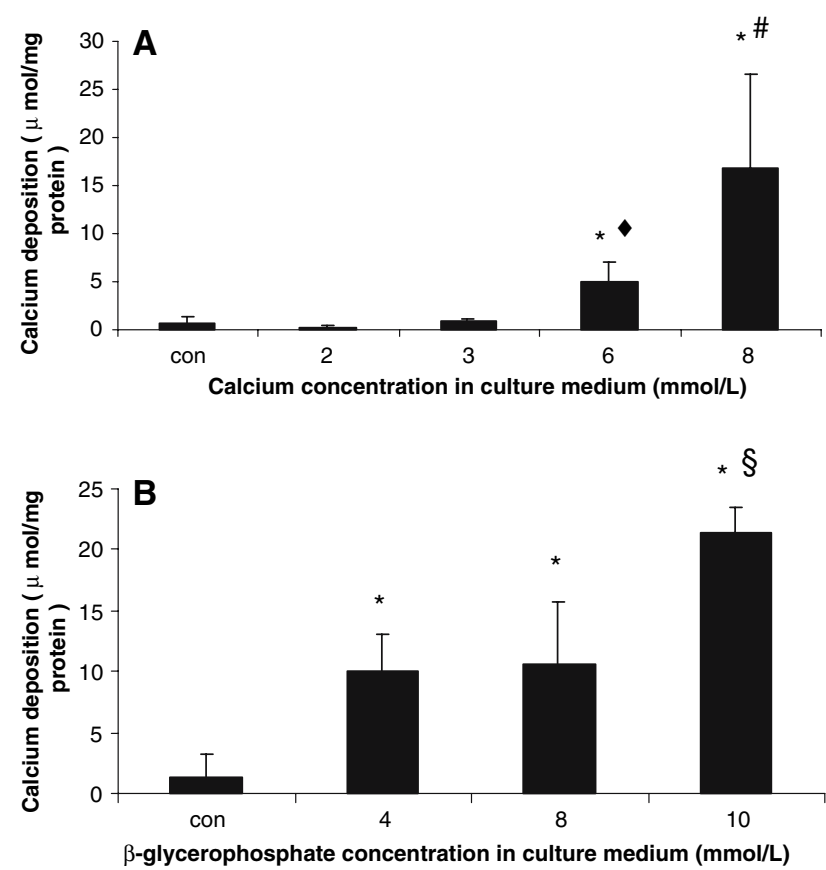

Fig. 2 (A) Dose-dependent effects of $\mathrm{CaCl}_{2}$ on calcification of neonatal rat VSMCs. (B) Dose-dependent effects of $\beta$-glycerophosphate on calcification of neonatal rat VSMCs. VSMCs were treated for 21 days with calcification medium containing varying concentrations of $\mathrm{Ca}^{2+}$ ions or $\beta$-glycerophosphate. Control cultures $(=\mathrm{con})$ were incubated with DMEM, $10 \%$ FBS, and antibiotics. Calcium deposition was quantified by $o$-cresolphthalein method. The data are presented as mean \pm SEM $(n=3-9)$. $* P<0.05$ vs. control cultures. $\diamond P<0.05$ vs. 2 and $3 \mathrm{mM}$ calcium in culture medium. $\# P<0.05$ vs. all other treatments

vascular calcification, the amount of calcium deposition was positively correlated to the $\beta$-glycerophosphate concentration in the calcification medium $(P<0.01$; Fig. $2 \mathrm{~B})$.

Calcium deposition and dependence of extracellular dexamethasone concentration

To determine the contribution of added dexamethasone, we incubated the VSMCs with various concentrations of dexamethasone (10-1,000 nmol/l). No significant differences in calcium deposition were observed between the cells incubated with $0,10,100$, and $1,000 \mathrm{nmol} / 1$ dexamethasone (Fig. 3A).

Calcium deposition and dependence of extracellular ascorbic acid concentration

To determine the contribution of ascorbic acid to calcium deposition, we incubated VSMCs with various concentrations of ascorbic acid $(0,5,50$, and $500 \mu \mathrm{g} / \mathrm{ml})$. At the highest concentration $(500 \mu \mathrm{g} / \mathrm{ml})$ ascorbic acid was associated with significantly more calcium deposition than
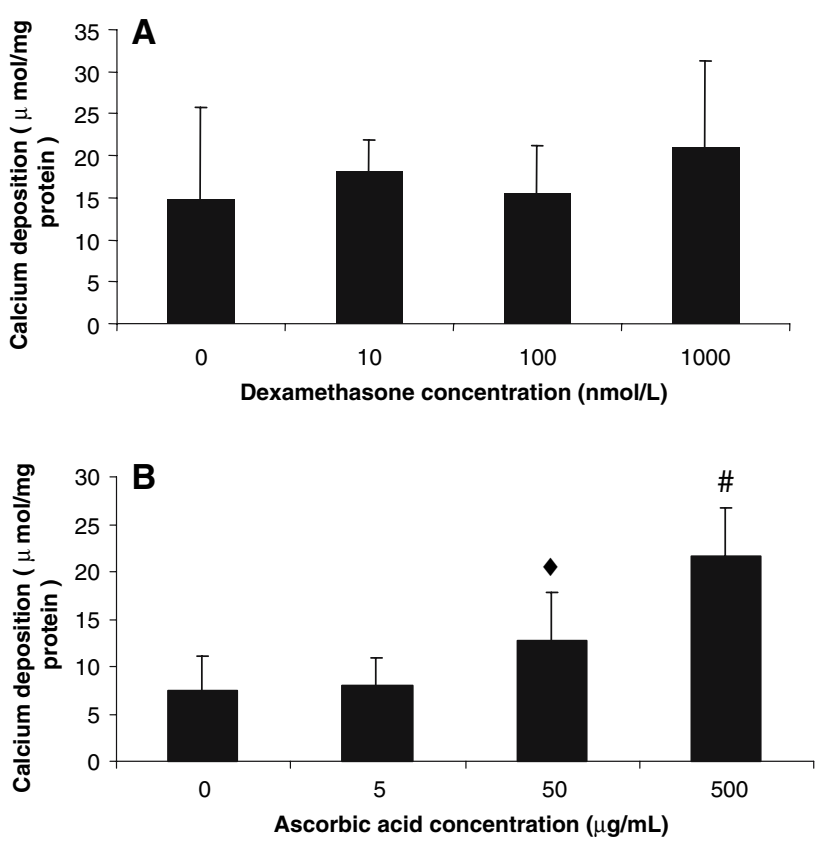

Fig. 3 (A) Dose-dependent effects of dexamethasone on calcification of neonatal rat VSMCs. (B) Dose-dependent effects of ascorbic acid on calcification of neonatal rat VSMCs. VSMCs were treated for 21 days with calcification medium containing varying amounts of dexamethasone or ascorbic acid. Calcium deposition was quantified by $o$-cresolphthalein method. The data are presented as mean \pm SEM $(n=6-12)$. $\# P<0.05$ vs. all other treatments. $\diamond P<0.05$ vs. 0 and $5 \mu \mathrm{g} / \mathrm{ml}$ ascorbic acid

at all other concentrations (Fig. 3B). However, $500 \mu \mathrm{g} / \mathrm{ml}$ ascorbic acid caused a considerable decrease in $\mathrm{pH}$ of the culture medium. Since calcium deposition was positively correlated with ascorbic acid concentration $(P<0.01)$, we decided to use $50 \mu \mathrm{g} / \mathrm{ml}$ ascorbic acid in future calcification studies, the highest concentration of ascorbic acid that did not cause acidification of the medium.

\section{Effects of amlodipine on in vitro VSMC calcification}

To study the effect of the CA amlodipine on VSMC calcification, VSMCs were incubated for 2-3 weeks with calcification medium supplemented with various concentrations of amlodipine $(0.01-1 \mu \mathrm{mol} / \mathrm{l})$. Incubation of neonatal rat VSMCs with amlodipine had no effect on VSMC calcification, at none of the concentrations tested (Fig. 4A).

Effects of atorvastatin on in vitro VSMC calcification

To study the effect of the statin atorvastatin on VSMC calcification, VSMCs were incubated for 2-3 weeks with calcification medium supplemented with various concentrations of atorvastatin $(2-50 \mu \mathrm{mol} / \mathrm{l})$. Atorvastatin 

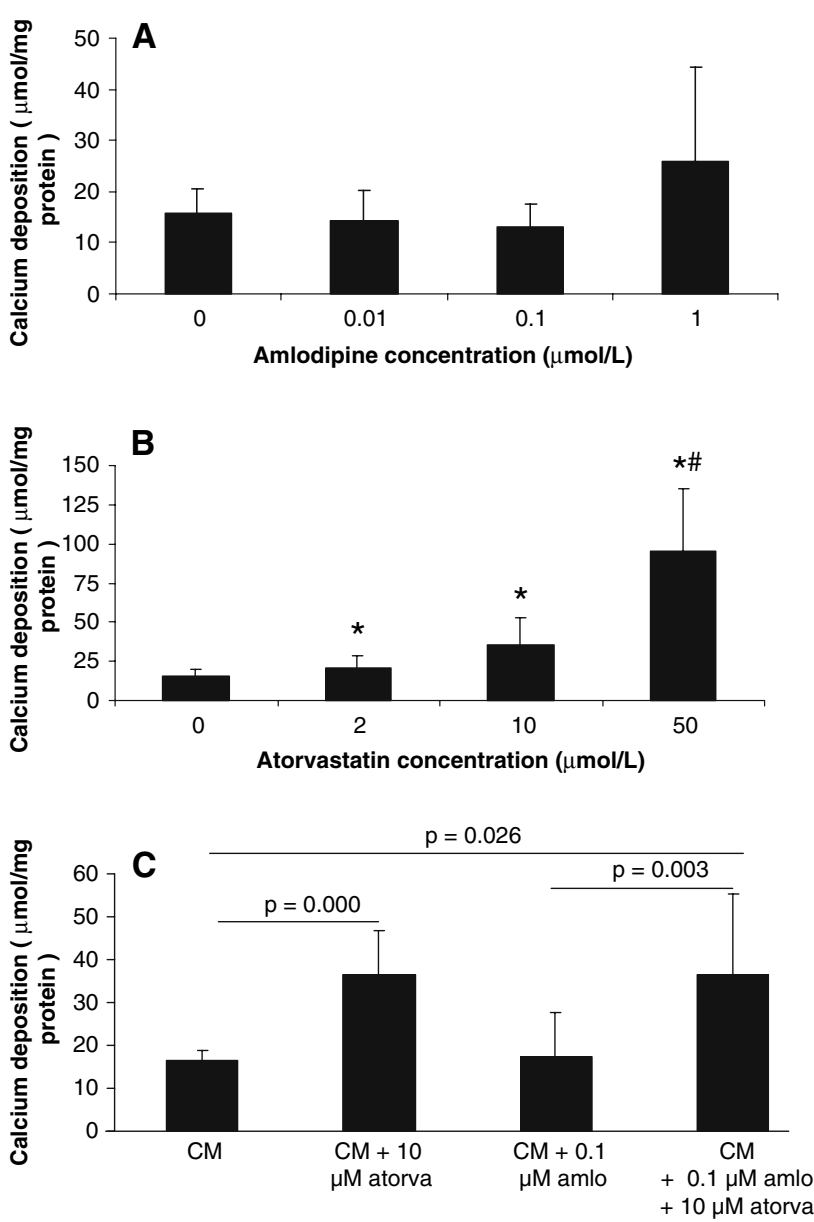

Fig. 4 (A) Dose-dependent effects of amlodipine on calcification of neonatal rat VSMCs. (B) Dose-dependent effects of atorvastatin on calcification of neonatal rat VSMCs. (C) Effects of amlodipine, atorvastatin and a combination of both treatments on neonatal rat VSMC calcification. VSMCs were treated for 21 days with calcification medium containing varying concentrations of atorvastatin or amlodipine, a combination of both, or none of them (control). Calcium deposition was quantified by $o$-cresolphthalein method. The data are presented as mean $\pm \operatorname{SEM}(n=15) . * P<0.05$ when compared to untreated control cultures. $\# P<0.05$ when compared to all other treatments

increased VSMC calcification dose-dependently (Fig. 4B). At a concentration of $2 \mu \mathrm{mol} / \mathrm{l}$ atorvastatin, calcium deposition was increased by $30 \%(P=0.04)$ when compared to VSMCs incubated with atorvastatin-free calcification medium. At concentrations of 10 and $50 \mu \mathrm{mol} / \mathrm{l}$ atorvastatin, calcium deposition was increased 2.3-fold and 6.1-fold, respectively $(P<0.001)$ when compared to VSMCs incubated with atorvastatin-free calcification medium.

Effect of a combination of CA and statin on in vitro VSMC calcification

To study the effect of combining amlodipine and atorvastatin treatment on VSMC calcification, VSMCs were incubated for 2-3 weeks with calcification medium supplemented with $0.1 \mu \mathrm{mol} / \mathrm{l}$ amlodipine, $10 \mu \mathrm{mol} / \mathrm{l}$ atorvastatin, or a combination of these drugs in the same concentrations. Incubation of VSMCs with $0.1 \mu \mathrm{mol} / 1$ amlodipine had no effect on VSMC calcification. Incubation of VSMCs with $10 \mu \mathrm{mol} / \mathrm{l}$ atorvastatin resulted in a 2.2-fold increased calcium deposition when compared to control cultures treated with calcification medium only (Fig. 4C) $(P<0.001)$. Treatment with a combination of amlodipine and atorvastatin also resulted in a 2.2-fold increased calcium deposition when compared to control cultures treated with calcification medium only $(P=0.026)$. The combination therapy resulted in significantly more calcium deposition than treatment with amlodipine alone $(P=0.003)$, and as much calcium deposition compared to treatment with atorvastatin alone (n.s.).

Effects of amlodipine, atorvastatin, and their combination on VSMC proliferation

VSMC proliferation was assessed at day 4 and day 9. After 4 days of incubation in calcification medium supplemented with amlodipine $(1 \mu \mathrm{mol} / \mathrm{l})$, VSMC proliferation was decreased by $21 \%(P<0.001)$ as compared to proliferation of VSMCs cultured in amlodipine-free calcification medium (Fig. 6A, online supplement). After 9 days of incubation, VSMC proliferation had increased by $14 \%$ $(P<0.05)$ when treated with $0.01 \mu \mathrm{mol} / 1$ amlodipine, but at an amlodipine concentration of $1 \mu \mathrm{mol} / \mathrm{l}$, VSMC proliferation had decreased by $26 \%(P<0.01)$ as compared to proliferation of VSMCs cultured in amlodipine-free calcification medium (Fig. 5A). Atorvastatin, at a concentration of $50 \mu \mathrm{mol} / \mathrm{l}$, decreased VSMC proliferation by $50 \%$ $(P<0.001$; Fig. 5B). Treatment of VSMCs with the combination of $0.1 \mu \mathrm{mol} / 1$ amlodipine and $10 \mu \mathrm{mol} / \mathrm{l}$ atorvastatin did not result in significant changes in cell proliferation compared to VSMCs incubated in the absence of these drugs.

Effects of amlodipine, atorvastatin, and their combination on VSMC apoptosis

VSMCs incubated in calcification medium only and VSMCs incubated with amlodipine $(0.01-1 \mu \mathrm{mol} / \mathrm{l})$ or atorvastatin $(2$ and $10 \mu \mathrm{mol} / \mathrm{l})$ in calcification medium for $72 \mathrm{~h}$ hardly contained any apoptotic nuclei. Amlodipine at the highest concentration tested $(1 \mu \mathrm{mol} / \mathrm{l})$, caused apoptosis of on average $0.05 \%$ of the cells (n.s. compared to control). At the highest concentration of atorvastatin $(50 \mu \mathrm{mol} / \mathrm{l})$, however, $5.6 \%$ of the nuclei were apoptotic $(P<0.05$ vs. control, 2 and $10 \mu \mathrm{mol} / 1$ atorvastatin; Fig. 6$)$. 

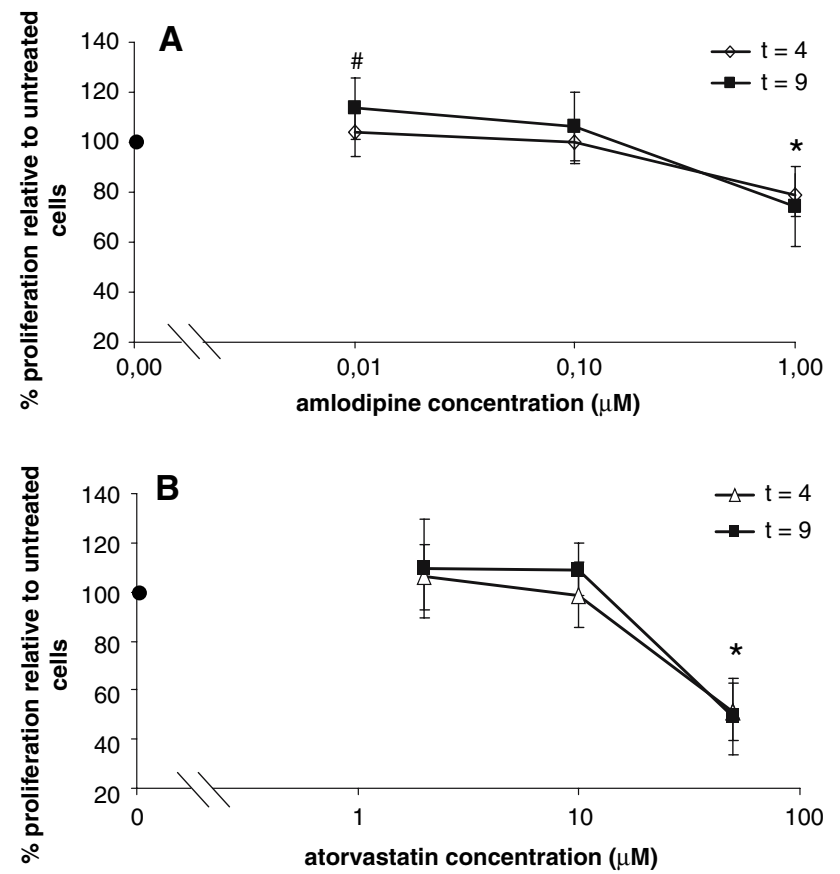

Fig. 5 Effect of amlodipine and atorvastatin on VSMC proliferation. VSMC proliferation was assessed at day 4 and day 9 using the Cell Proliferation Kit II (XTT)(Roche). VSMC proliferation is depicted as percentage relative to untreated cells $(=100 \%)$ (A) Effect of amlodipine. (B) Effect of atorvastatin. \#P<0.05 vs. control cultures. $* P<0.05$ vs. control cultures

VSMCs treated with the combination of $0.1 \mu \mathrm{mol} / 1$ amlodipine and $10 \mu \mathrm{mol} / \mathrm{l}$ atorvastatin for $72 \mathrm{~h}$ had a number of apoptotic nuclei that did not significantly differ from those observed in $10 \mu \mathrm{mol} / \mathrm{l}$ atorvastatin only,
$0.1 \mu \mathrm{mol} / 1$ amlodipine only, and VSMCs incubated in calcification medium in the absence of these drugs.

\section{Discussion}

In an in vitro model of vascular calcification of rat aortic VSMCs, calcium deposition was dependent upon the extracellular concentration of organic phosphate $(P<0.01)$, calcium ions $(P<0.01)$, and ascorbic acid $(P<0.01)$. While studying the effect of the CA amlodipine and the statin atorvastatin, alone and in combination, on calcification, we found that, at none of the concentrations tested $(0.01-1 \mu \mathrm{mol} / \mathrm{l})$ did amlodipine have any effect on VSMC calcification in this model, nor on development of apoptosis. In contrast, atorvastatin stimulated VSMC calcification at a concentration of $\geq 10 \mu \mathrm{mol} / \mathrm{l}(P<0.05)$, and $50 \mu \mathrm{mol} / \mathrm{l}$ inhibited VSMC proliferation and induced apoptosis $(P<0.05)$.

VSMCs retain remarkable plasticity, even in adult animals. VSMCs can undergo rapid and reversible changes in its phenotype in response to changes in local environmental conditions [22]. Due to production of a variety of cytokines by subendothelial macrophages, proliferation, and migration of VSMCs to the intimal layer can occur, thereby contributing to the process of intimal thickening and atherosclerosis.

Neonatal VSMCs resemble VSMCs in atherosclerotic plaque, since they retain the proliferative phenotype. Neonatal aortic explants exhibit rapid outgrowth of VSMCs, and we chose to use these cells to develop a model
Fig. 6 VSMCs were treated with $1 \mu \mathrm{mol} / \mathrm{l}$ amlodipine $(\mathbf{A}, \mathbf{B})$ or $50 \mu \mathrm{mol} / 1$ atorvastatin $(\mathbf{C}, \mathbf{D})$ for $72 \mathrm{~h}$. Nuclei were visualized with Hoechst 33342. Apoptotic nuclei are circled in panel $\mathrm{C}$ and indicated with arrows in panel D. Microscopy: magnification A and $\mathrm{C} \times 100, \mathrm{~B}$ and $\mathrm{D} \times 400$
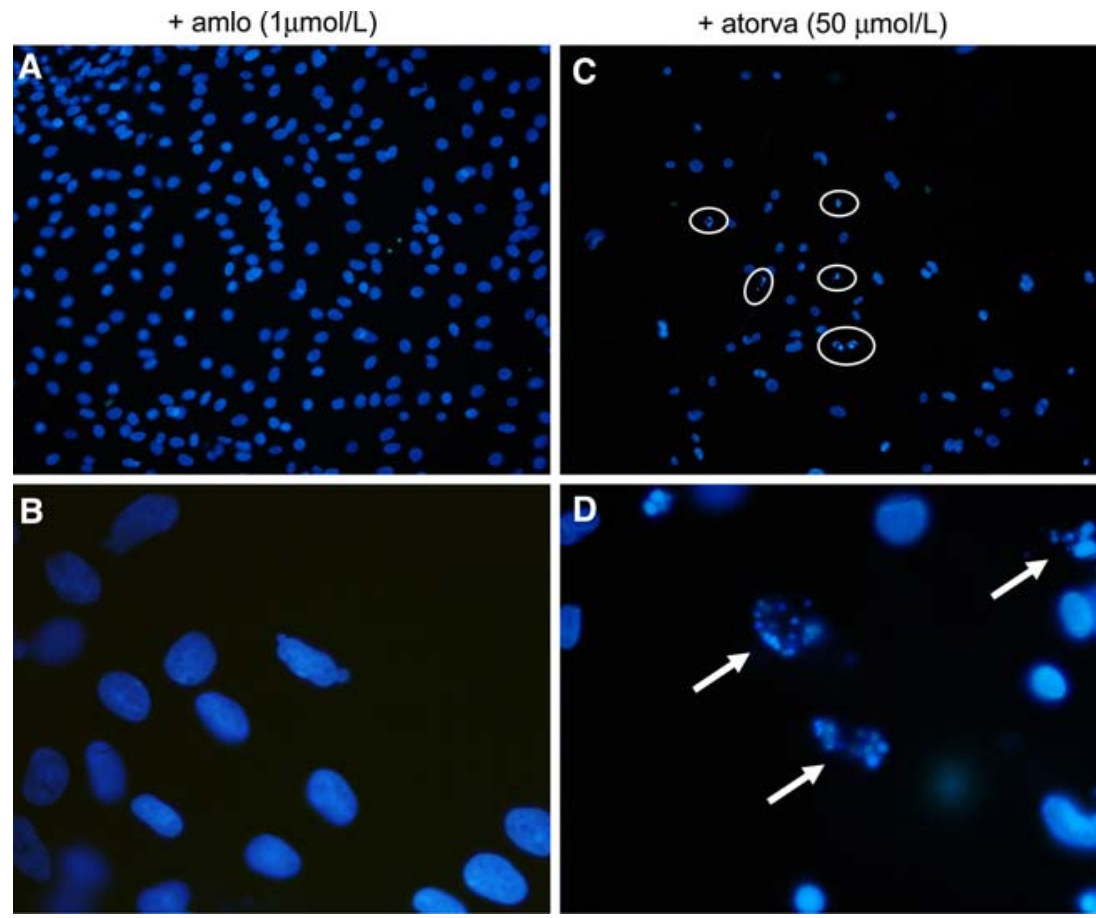
of vascular calcification. VSMCs were shown to migrate from the aortic explant within several days, and were identified as VSMCs by immunohistochemistry [8].

Dialysis patients have accelerated atherosclerosis, with extensive calcification of both the intima and the media [23]. Hyperphosphatemia has been implicated in this process [24]. Inorganic phosphate $\left(\mathrm{P}_{\mathrm{i}}\right)$ levels have previously been shown to regulate human aortic smooth muscle cell (HSMC) culture mineralization in vitro [25, 26]. In the present study, the amount of calcium deposition in neonatal rat VSMCs correlated positively to the $\beta$-glycerophosphate concentration in the calcification medium.

In the present study, the amount of calcium deposition in rat aortic VSMCs correlated to the calcium concentration in the calcification medium. This observation was not a result of passive calcium deposition, since incubation of VSMCs with normal growth medium supplemented with $8 \mathrm{mmol} / \mathrm{C} \mathrm{CaCl}_{2}$ (final concentration) did not result in calcium deposition by these cells.

Shioi et al. [21] have previously demonstrated that culturing bovine VSMCs in the presence of $\beta$-glycerophosphate, ascorbic acid, and insulin can induce diffuse calcification in a manner analogous to in vitro mineralization of osteoblasts. The present study also showed that $\beta$-glycerophosphate is crucial for the induction of calcification in rat aortic VSMCs. Ascorbic acid augments this process. Dexamethasone was demonstrated to be less vital in this model of aortic VSMC calcification.

Statins possess potent lipid-lowering effects. Besides, statins exert pleiotropic effects on vascular wall cells, which include improvement of endothelial function, stabilization of the atherosclerotic plaque, and suppression of inflammation [27, 28]. Although several studies have failed to demonstrate an effect of statins on the progression of calcific aortic stenosis [29, 30], statins have also been shown to decrease the progression of coronary artery calcification and aortic valve calcification [17, 18, 31, 32]. Accordingly, Kizu et al. have demonstrated that statins inhibit calcification in an in vitro model of inflammatory vascular calcification [33]. Using interferon- $\gamma, 1 \alpha, 25$ dihydroxyvitamin $\mathrm{D}_{3}$, tumor necrosis factor- $\alpha$, and oncostatin $\mathrm{M}$ to induce calcification in human VSMCs, it was demonstrated that cerivastatin and atorvastatin inhibited calcification dose-dependently. However, one should realize that this model differs from the model we have used in the present study. Whereas Kizu et al. used inflammatory mediators to induce calcification of the VSMCs, we used increased levels of $\mathrm{CaCl}_{2}$ and organic phosphate in the culture medium to promote calcification. Statins are known to have anti-inflammatory effects, and therefore, their inhibitory effect on calcification induced by inflammatory mediators is to be expected. In addition, low doses of statins have also been shown to protect human aortic
VSMCs from inorganic phosphate-induced calcification [34].

In contrast to the aforementioned studies, the present study showed a dose-dependent stimulatory effect of atorvastatin $(10$ and $50 \mu \mathrm{mol} / \mathrm{l})$ on calcification of VSMCs incubated in calcification medium. However, the dose of atorvastatin used in the present study was much higher than in the study described by Son et al. (500x higher at the highest dose) [34], and most likely explains the discrepancy between the results obtained by Son et al. and the results of the present study.

Reynolds et al. [35] have demonstrated that human VSMCs undergo vesicle-mediated calcification in response to changes in calcium and phosphate concentrations in the culture medium. Elevated calcium and phosphate concentrations resulted in increased release of vesicles and stimulation of apoptosis. Calcification was initiated by release of membrane-bound matrix vesicles from living cells and also by apoptotic bodies from dying cells. Vesicles released by VSMCs after prolonged exposure to calcium and phosphate contained preformed basic calcium phosphate, and calcified extensively. The present study confirms that statins stimulate apoptosis of VSMCs. Apoptotic bodies can calcify extensively [36], so a likely mechanism of induction of calcification by atorvastatin is through induction of apoptosis. Indeed, atorvastatin stimulated both apoptosis and calcification in our model.

Myocardial infarction and unstable angina usually arise from the disruption of mildly stenosed atherosclerotic lesions. Such vulnerable plaques typically contain a large amount of lipid and have a preponderance of inflammatory cells at the shoulders of the plaque and a thin fibrous cap. Calcium is found infrequently in the culprit lesion of ruptured plaque. Thus, plaque vulnerability is not associated with size, but with composition. Beckman et al. [37] presented the concept "that calcium is not a critical substrate for plaque disruption and is, in fact, associated with more stable plaques." Therefore, statin-induced alterations in the plaque's composition from lipid to fibrous tissue without or with calcification would decrease the plaque's vulnerability to rupture, thereby decreasing the risk of acute coronary syndromes.

Prolonged intensive lipid-lowering has been demonstrated to increase calcium content of plaques as assessed by MRI in vivo, and lowered plaque lipid content at about the same extent, without a pronounced effect on plaque size [38]. Since atherosclerotic plaque composition plays an important role in plaque stability, with lipids destabilizing the plaque and calcification stabilizing the plaque, the effect of statins on plaque composition may be favorable. In addition to lipid-lowering and calcificationinducing effects, we observed that statins have an anti-proliferative effect on VSMCs. Less proliferation of VSMCs may destabilize the plaque. However, this only 
occurred at high doses of atorvastatin, whereas calcification induction occurred at lower dose of atorvastatin.

Although several in vitro and in vivo studies have demonstrated that combining $\mathrm{CA}$ and statin therapy might be more atheroprotective than either treatment alone [19, 20, 39], the present study found no difference between the combination of amlodipine and atorvastatin therapy, and atorvastatin therapy alone on VSMC calcification.

In conclusion, extracellular $\beta$-glycerophosphate and $\mathrm{CaCl}_{2}$ concentrations are important determinants of in vitro calcification of rat aortic VSMCs. Ascorbic acid stimulates this process. Dexamethasone was demonstrated to be less vital in this model of VSMC calcification. In vitro calcification of rat aortic VSMCs is not affected by amlodipine treatment, but is stimulated by atorvastatin treatment. As high concentrations of atorvastatin have a pro-apoptotic effect, the pro-calcification effect of atorvastatin may be explained by the production of apoptotic bodies that act as foci for calcium deposition. The latter finding may explain the plaque-stabilizing effect reported for statins.

\section{References}

1. Detrano RC, Wong ND, Doherty TM et al (1997) Prognostic significance of coronary calcific deposits in asymptomatic highrisk subjects. Am J Med 102:344-349

2. Devries S, Wolfkiel C, Fusman B et al (1995) Influence of age and gender on the presence of coronary calcium detected by ultrafast computed tomography. J Am Coll Cardiol 25:76-82

3. Goodman WG, Goldin J, Kuizon BD et al (2000) Coronaryartery calcification in young adults with end-stage renal disease who are undergoing dialysis. N Engl J Med 342:1478-1483

4. Wagenknecht LE, Bowden DW, Carr JJ et al (2001) Familial aggregation of coronary artery calcium in families with type 2 diabetes. Diabetes 50:861-866

5. Anderson HC (1983) Calcific diseases. A concept. Arch Pathol Lab Med 107:341-348

6. Bostrom K, Watson KE, Horn S et al (1993) Bone morphogenetic protein expression in human atherosclerotic lesions. J Clin Invest 91:1800-1809

7. Mohler ER III, Gannon F, Reynolds C et al (2001) Bone formation and inflammation in cardiac valves. Circulation 103: $1522-1528$

8. Trion A, van der Laarse A (2004) Vascular smooth muscle cells and calcification in atherosclerosis. Am Heart J 147:808-814

9. Nayler WG (1999) Review of preclinical data of calcium channel blockers and atherosclerosis. J Cardiovasc Pharmacol 33(Suppl 2):S7-S11

10. Tulenko TN, Sumner AE, Chen M et al (2001) The smooth muscle cell membrane during atherogenesis: a potential target for amlodipine in atheroprotection. Am Heart J 141:S1-11

11. Betz E, Weiss HD, Heinle $\mathrm{H}$ et al (1991) Calcium antagonists and atherosclerosis. J Cardiovasc Pharmacol 18(Suppl 10): S71-S75

12. Waters D, Lesperance J, Francetich M et al (1990) A controlled clinical trial to assess the effect of a calcium channel blocker on the progression of coronary atherosclerosis. Circulation 82:1940-1953

13. Mak IT, Boehme P, Weglicki WB (1992) Antioxidant effects of calcium channel blockers against free radical injury in endothelial cells. Correlation of protection with preservation of glutathione levels. Circ Res 70:1099-1103

14. Eickelberg O, Roth M, Block LH (1997) Effects of amlodipine on gene expression and extracellular matrix formation in human vascular smooth muscle cells and fibroblasts: implications for vascular protection. Int J Cardiol 62(Suppl 2):S31-S37

15. Parhami F, Morrow AD, Balucan J et al (1997) Lipid oxidation products have opposite effects on calcifying vascular cell and bone cell differentiation. A possible explanation for the paradox of arterial calcification in osteoporotic patients. Arterioscler Thromb Vasc Biol 17:680-687

16. Pohle K, Maeffert R, Ropers D et al (2001) Progression of aortic valve calcification. Association with coronary atherosclerosis and cardiovascular risk factors. Circulation 104:1927-1932

17. Achenbach S, Ropers D, Pohle K et al (2002) Influence of lipidlowering therapy on the progression of coronary artery calcification: a prospective evaluation. Circulation 106:1077-1082

18. Shavelle DM, Takasu J, Budoff MJ et al (2002) HMG CoA reductase inhibitor (statin) and aortic valve calcium. Lancet 359:1125-1126

19. Jukema JW, Zwinderman AH, van Boven AJ et al (1996) Evidence for a synergistic effect of calcium channel blockers with lipid-lowering therapy in retarding progression of coronary atherosclerosis in symptomatic patients with normal to moderately raised cholesterol levels. The REGRESS Study Group. Arterioscler Thromb Vasc Biol 16:425-430

20. Leibovitz E, Beniashvili M, Zimlichman R et al (2003) Treatment with amlodipine and atorvastatin have additive effect in improvement of arterial compliance in hypertensive hyperlipidemic patients. Am J Hypertens 16:715-718

21. Shioi A, Nishizawa Y, Jono S et al (1995) Beta-glycerophosphate accelerates calcification in cultured bovine vascular smooth muscle cells. Arterioscler Thromb Vasc Biol 15:2003-2009

22. Schwartz SM, Campbell GR, Campbell JH (1986) Replication of smooth muscle cells in vascular disease. Circ Res 58:427-444

23. Locatelli F, Cannata-Andia JB, Drueke TB et al (2002) Management of disturbances of calcium and phosphate metabolism in chronic renal insufficiency, with emphasis on the control of hyperphosphataemia. Nephrol Dial Transplant 17:723-731

24. Shioi A, Taniwaki H, Jono S et al (2001) Monckeberg's medial sclerosis and inorganic phosphate in uremia. Am J Kidney Dis 38:S47-S49

25. Giachelli CM, Jono S, Shioi A et al (2001) Vascular calcification and inorganic phosphate. Am J Kidney Dis 38:S34-S37

26. Jono S, McKee MD, Murry CE et al (2000) Phosphate regulation of vascular smooth muscle cell calcification. Circ Res 87:E10 E17

27. Kwak BR, Mulhaupt F, Mach F (2003) Atherosclerosis: antiinflammatory and immunomodulatory activities of statins. Autoimmun Rev 2:332-338

28. Takemoto M, Liao JK (2001) Pleiotropic effects of 3-hydroxy-3methylglutaryl Coenzyme A reductase inhibitors. Arterioscler Thromb Vasc Biol 21:1712-1719

29. Wanner C, Krane V, Marz W et al (2006) Atorvastatin in patients with type 2 diabetes mellitus undergoing hemodialysis. N Engl J Med 353:238-248

30. Cowell SJ, Newby DE, Prescott RJ et al (2005) A randomized trial of intensive lipid-lowering therapy in calcific aortic stenosis. N Engl J Med 352:2389-2397

31. Novaro GM, Tiong IY, Pearce GL et al (2001) Effect of hydroxymethylglutaryl coenzyme A reductase inhibitors on 
the progression of calcific aortic stenosis. Circulation 104: 2205-2209

32. Callister TQ, Raggi P, Cooil B et al (1998) Effect of HMG-CoA reductase inhibitors on coronary artery disease as assessed by electron-beam computed tomography. N Engl J Med 339: 1972-1978

33. Kizu A, Shioi A, Jono S et al (2004) Statins inhibit in vitro calcification of human vascular smooth muscle cells induced by inflammatory mediators. J Cell Biochem 93:1011-1019

34. Son B, Kozaki K, Iijima K et al (2006) Statins protect human aortic smooth muscle cells from inorganic phosphate-induced calcification by restoring Gas6-Axl survival pathway. Circ Res 98:1024-1031

35. Reynolds JL, Joannides AJ, Skepper JN et al (2004) Human vascular smooth muscle cells undergo vesicle-mediated calcification in response to changes in extracellular calcium and phosphate concentrations: a potential mechanism for accelerated vascular calcification. J Am Soc Nephrol 15:2857-2867
36. Proudfoot D, Skepper JN, Hegyi L et al (2000) Apoptosis regulates human vascular calcification in vitro: evidence for initiation of vascular calcification by apoptotic bodies. Circ Res 87:1055-1062

37. Beckman JA, Ganz J, Craeger MA et al (2001) Relationship of clinical presentation and calcification of culprit coronary artery stenoses. Arterioscler Thromb Vasc Biol 21:1618-1622

38. Zhao XQ, Yuan C, Hatsukami TS et al (2001) Effects of prolonged intensive lipid-lowering therapy on the characteristics of carotid atherosclerotic plaques in vivo by MRI: a case-control study. Arterioscler Thromb Vasc Biol 21:1623-1629

39. Anon (2003) Effect of nifedipine and cerivastatin on coronary endothelial function in patients with coronary artery disease: the ENCORE I Study (Evaluation of Nifedipine and Cerivastatin On Recovery of coronary Endothelial function). Circulation 107:422-428 